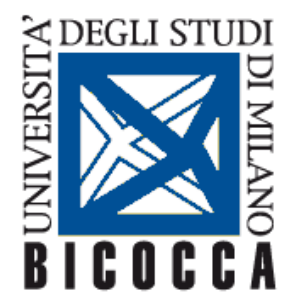

University of Milano-Bicocca

School of Law

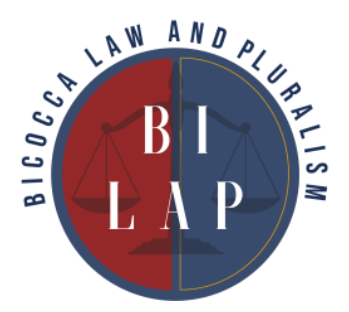

Costituzionalismo e diversità etnica: il caso della Bosnia-Erzegovina

\author{
Edin Skrebo
}

University of Milan-Bicocca School of Law Research Paper Series No. 20-05

https://giurisprudenza.unimib.it 


\title{
Costituzionalismo e diversità etnica: il caso della Bosnia-Erzegovina Constitutionalism and ethnic diversity: the case of Bosnia and Herzegovina
}

Edin Skrebo

(Dottorando di ricerca in Scienze giuridiche, curriculum Law and Pluralism - Università degli Studi di Milano-

Bicocca)

\begin{abstract}
Il presente contributo cerca di illustrare - prendendo spunto dal venticinquesimo anniversario dall'entrata in vigore della Costituzione della Bosnia-Erzegovina - il ruolo centrale che il fattore etnico assume all'interno dell'ordinamento bosniaco. Il testo si apre dedicando alcune considerazioni introduttive alla peculiare modalità con cui questa Carta costituzionale è stata redatta e adottata. Nella parte centrale del contributo, invece, si è posta l'attenzione sull'"istituzionalizzazione del fattore etnico", il quale assume tanto peso circa l'assetto federale dello Stato, quanto per il funzionamento e la formazione delle istituzioni della Bosnia. Da ultimo, sono stati evidenziati alcuni limiti e criticità emersi dall'ordinamento costituzionale del paese.
\end{abstract}

Abstract: Twenty-five years have passed since the new Constitution of Bosnia and Herzegovina came into force. To celebrate this anniversary, the present article will try to highlight its peculiarities: first of all, the way in which this Constitution was drafted and adopted. Instead, the central part of the paper will be aimed at an in-depth analysis of the constitutional text on the topic of ethnic diversity. What emerges is a constitutional order based on the institutionalization of ethnic diversity, both at the level of the local structure of the States, and at the level of the composition and functioning of the state institutions themselves. In conclusion, the article will focus on a brief illustration of the main issues that have emerged over the last few years.

SOMMARIO: 1. Introduzione - 2. Pace e Costituzione: tutte le deviazioni del costituzionalismo alla luce del principio della pace? - 3. La gestione della diversità etnica a livello locale, ovvero del "federalismo etnico" -4. L'istituzionalizzazione del fattore etnico all'interno dell'ordinamento bosniaco - 4.1. Il potere legislativo - 4.2. Il potere esecutivo - 4.3. Il potere giudiziario - 5. Limiti e futuro del costituzionalismo bosniaco

\section{Introduzione}

Il 14 dicembre del 2020 si è celebrato il venticinquesimo anniversario dalla firma del General Framework Agreement for a Peace in Bosnia and Herzegovina, forse meglio noto come Accordo di pace di Dayton, che pose fine alla sanguinosa guerra iniziata nel 1992. Contemporaneamente, però, si celebra anche l'entrata in vigore della nuova Costituzione della Bosnia-Erzegovina, quale parte (Annex IV) dell'Accordo.

Una caratteristica intrinseca che gli anniversari possiedono è quella di rappresentare un'occasione per dedicarsi ad alcune riflessioni più approfondite e, se possibile, ad un bilancio. È proprio prendendo spunto da una tale occasione che si vorrebbe perciò tentare, per sommi capi e senza pretese di esaustività, di illustrare al lettore quali siano le caratteristiche della Costituzione bosniaca e dell'ordinamento che ne è derivato. Più in particolare, si dedicherà attenzione a quelle che sono state le soluzioni adottate per gestire, da un punto di vista istituzionale, la molteplicità etnica che contraddistingue il paese, cercando, non solo di evidenziare e spiegare il ruolo assunto dall'etnicità all'interno dell'ordinamento costituzionale bosniaco, ma anche di far emergere luci ed ombre di questa evoluzione. Non prima, però, di aver illustrato il particolare contesto che ha dato $\mathrm{i}$ natali a questa Costituzione, le cui peculiarità sarebbe difficile comprendere senza le dovute premesse. 


\section{Pace e Costituzione: tutte le deviazioni del costituzionalismo alla luce del principio della pace?}

In apertura è stato ricordato che la Costituzione della Bosnia-Erzegovina si contraddistingue per essere un allegato, precisamente l'Allegato IV, dell'Accordo di Dayton. Come tale, il testo si caratterizza per essere un esempio di Costituzione eterodiretta, in quanto frutto in primo luogo del lavoro e delle soluzioni elaborate dai negoziatori statunitensi e della Comunità europea, guidati dal Vicesegretario di Stato statunitense Richard Holbrooke. È proprio soffermandosi su questo momento generativo della Costituzione che si può scorgere la prima peculiarità di questo testo. Infatti, la redazione e l'entrata in vigore della Carta costituzionale sembrano seguire modalità alquanto «insolite» ${ }^{1}$. Prima di tutto, il testo non risulta essere stato redatto da un' apposita assemblea costituente o dall'Assemblea parlamentare della Bosnia ${ }^{2}$, ma trae le proprie origini da una serie di accordi tra $\mathrm{i}$ ministri degli esteri della Bosnia, Croazia e Serbia ${ }^{3}$ ed i negoziatori della Comunità internazionale. I primi due accordi, che poi avrebbero costituito l'ossatura dell'Annex IV del General Framework Agreement for Peace in materia costituzionale e sull'assetto territoriale da conferire al paese, sono stati sottoscritti in data 8 settembre 1995 a Ginevra ${ }^{4}$ ed il 26 settembre del medesimo anno a New York $^{5}$. Con questi primi due atti, definiti "Accordi sui principi base", è stato possibile porre «le basi per la ricostruzione istituzionale ed economica dello Stato» ${ }^{6}$.

Inoltre, l'entrata in vigore della Costituzione non è stata preceduta da alcuna forma di ratifica da parte della popolazione attraverso un referendum approvativo, né vi è stata una ratifica da parte dell'Assemblea parlamentare della Bosnia-Erzegovina. Infatti, il dettato costituzionale, in modo alquanto laconico, prevede che l'entrata in vigore della Costituzione avvenga «upon signature of the General Framework Agreement ${ }^{7}$.Tale modalità trova spiegazione nel semplice fatto che la Costituzione si caratterizza per essere una parte (Allegato) dell'Accordo di pace e come tale necessariamente produce i suoi effetti dalla ratifica del General Framework Agreement. A questo si aggiunge anche il fatto che il testo costituzionale, sempre all'articolo XII, definisce la nuova Costituzione come un atto «amending and superseding the Constitution of The Republic of Bosnia and Herzegovina», riferendosi al precedente testo della Costituzione adottata nel 1993. In realtà, quello che ne emerge è uno scostamento palese rispetto alla procedura prevista dalla Costituzione del 1993, in materia di revisione costituzionale. Infatti, l'articolo 268 sanciva che le proposte di revisione costituzionale venissero redatte dall'Assemblea parlamentare in seduta comune e che, dopo un dibattito pubblico di alcune settimane, il testo venisse votato dalla medesima Assemblea riunita in seduta comune. Si prevedeva, inoltre, il quorum deliberativo di due terzi dei membri dell'Assemblea per l'approvazione dell'emendamento alla Costituzione. Ne emerge che - pur avendo i costituenti del 1995 adottato il termine "emendamento" per il nuovo testo rispetto a quello precedente - in realtà ci troviamo di fronte ad una nuova Costituzione che non si limita meramente a modificare alcuni aspetti dell'ordinamento costituzionale precedente, ma ne instaura uno completamente nuovo.

\footnotetext{
${ }^{1}$ S. Yee, The New Constitution of Bosnia and Herzegovina, in «European Journal of International Law», n. 7, 1996, p. 177.

${ }^{2}$ Ivi, pp. 178-180.

${ }^{3}$ La Bosnia era rappresentata dal Ministro Šaćirbegović, la Croazia da Granić e la Serbia da Milutinović.

${ }^{4}$ Il testo completo dell'Accordo è consultabile al seguente link: http://www.ohr.int/ohr_archive/ohr-principles-geneva-8sept-95/.

${ }^{5}$ Il testo dell'Accordo è consultabile al seguente link: http://www.ohr.int/?ohr_archive=joint-statement-new-york-26sept-95.

${ }^{6}$ J. Woelk, La transizione costituzionale della Bosnia Erzegovina. Dall'ordinamento imposto allo Stato multinazionale sostenibile? CEDAM, Padova, 2008, p. 79.

${ }^{7}$ Articolo XII, comma 1, della Costituzione della Bosnia-Erzegovina.
} 
È stata proprio la particolare procedura con cui questa Costituzione è stata redatta ed adottata che ha spinto parte della dottrina ${ }^{8}$ ad esprimere dei dubbi circa la sua democraticità, tanto da parlare di una «undemocratic origin» ${ }^{9}$.

Per cercare di chiarire questa questione - senza la pretesa di fornire una risposta definitiva appare necessario prendere le mosse dal dato testuale, e precisamente dall'ultimo paragrafo del Preambolo della Costituzione, in cui si afferma che «Bosniacs, Croats and Serbs, as a constituent people (along with Others), and citizens of Bosnia and Herzegovina hereby determine the Constitution» ${ }^{10}$. Stando al mero dato testuale, la democraticità del procedimento di formazione della Costituzione deriverebbe dalla volontà formale dei tre popoli costitutivi della Bosnia di dotare lo Stato di una nuova Carta costituzionale. Tuttavia, appare chiaro che sostenere la democraticità del processo costituente sulla base di questo enunciato non risulta essere un argomento dirimente. In realtà, una giustificazione a supporto di una sia pur minima democraticità del procedimento può essere reperita in tre dichiarazioni, rese in calce all'Allegato IV, firmate dai rappresentanti delle due Entità, che avrebbero costituito la Bosnia, e dal Presidente della Repubblica di Bosnia-Erzegovina, con cui si dichiarava l'approvazione del testo costituzionale «on behalf of its constituent people and citizens». Con questa formula sembra esprimere una sorta di mandato popolare conferito ai firmatari dell'Accordo per la redazione e l'approvazione della Carta.

Parte della dottrina ${ }^{11}$, servendosi proprio di questo enunciato, ha ritenuto di poter trovare una certa forma di democraticità non tanto nel momento generativo della Costituzione, ma a valle, nelle dichiarazioni rese dai rappresentanti locali (Entità) e statali (Presidente), già eletti da quelli che con la promulgazione della Carta avrebbero preso il nome di popoli costitutivi.

Ad ogni modo, per chi scrive, la questione della democraticità non può essere esaminata con lo stesso metro di paragone di una Costituzione frutto del lavoro dei costituenti autoctoni. In primo luogo, perché la Carta bosniaca assume in sé una natura ancipite: quale Costituzione da una parte, ma anche quale allegato di un accordo di carattere internazionale. Inoltre, le condizioni storiche che hanno portato alla stesura di questo testo sono state tali da impedire un procedimento costituente maggiormente condiviso. Infatti, tanto nell'ottica dei diplomatici della Comunità internazionale, quanto in quella delle fazioni in lotta, la priorità era quella di raggiungere il prima possibile un accordo che fosse in grado di porre fine alle ostilità e che potesse ricostruire uno Stato unitario e multinazionale allo stesso tempo ${ }^{12}$. Questo spiega almeno in parte, se non del tutto, perché la democraticità del procedimento sia stata sacrificata sull'altare del valore della pace e della necessità. Rendendosi così necessario un operato celere e deciso, al fine di evitare un ennesimo fallimento della Comunità internazionale nel pacificare il paese.

Ad ogni modo, la discussione dottrinale sulla natura democratica di questa Costituzione deve comunque trovare un momento di confronto/bilanciamento con il legittimo operato (se non la natura stessa), delle organizzazioni internazionali e dei paesi che hanno reso possibile il raggiungimento di un accordo di pace, primi fra tutti gli Stati Uniti, l'Inghilterra e la Francia.

\footnotetext{
${ }^{8}$ Sul punto si veda almeno: S. Yee, The New Constitution, art. cit., pp. 176-192; P. Gaeta, The Dayton Agreement and International Law, in «European Journal of International Law», n. 7, 1996, pp. 149-150; R. Kuzmanović, Il Costituzionalismo della Bosnia-Erzegovina fra nuovo sistema mondiale e transizione, in S.Gambino (a cura di), Costituzionalismo europeo e transizioni democratiche, Giuffrè, Milano, 2003, pp. 211-228; R. Kuzmanović, Ustavno pravo, Apeiron, Banja Luka, 2011, pp. 116-118; S. Savić, Republika Srpska poslje Dejtona, Pravni Fakultet u Banja Luci, Banja Luka, 1999, pp. 78-80.

${ }^{9}$ S. Yee, The New Constitution, art. cit., p. 180.

${ }^{10}$ Questo enunciato sembra prende spunto dal famoso incipit «We the People» della Costituzione degli Stati Uniti d'America. Ciò dimostra che la fonte di ispirazione per la Costituzione della Bosnia si ritrova proprio nell'ambito della cultura giuridica anglosassone, in particolare quella elaborata negli Stati Uniti. Cfr. B. A. Ackerman, We the people. Foundations, Harvard University press, Cambridge, 1991.

${ }^{11}$ S. Yee, The New Constitution, art. cit., pp. 179-181.

${ }^{12}$ K. Trnka, Specifičnosti ustavnog uređenja Bosne i Hercegovine, in «Journal for Constitutional Theory and Philosophy of Law», n. 9, 2009, pp. 48-49.
} 
Ulteriore aspetto che qui rileva evidenziare, è quello riguardante i rapporti intercorrenti tra l'ordinamento costituzionale bosniaco ed il diritto internazionale e sovranazionale, soprattutto per quanto riguarda la difesa dei diritti umani e delle libertà fondamentali, anche alla stregua della duplice natura della Costituzione. In particolare, all'articolo II, secondo comma, della Costituzione viene sancito che la Convenzione europea per la salvaguardia dei diritti umani e delle libertà fondamentali (CEDU) e i suoi protocolli, «shall apply directly in Bosnia and Herzegovina», aggiungendo anche che «these shall have priority over all other law». Alla CEDU, con il primo allegato alla Costituzione, viene aggiunta un'intera serie di altre Convenzioni internazionali in materia di diritti umani e di libertà fondamentali che trovano diretta applicazione nell'ordinamento del paese ${ }^{13}$. Questo significa che queste ultime costituiscono parte integrante del testo costituzionale della Bosnia, sia in senso formale che sostanziale, oltre che possedere un ruolo gerarchicamente sopraelevato rispetto alle leggi dello Stato. Sempre su questo fronte, appare interessante riportare quanto sancito dall'articolo III, comma 3, (b), della Costituzione, per cui «the general principles of international law shall be an integral part of the law of Bosnia and Herzegovina and the Entities», a conferma e dimostrazione dello stretto legame tra diritto internazionale e ordinamento bosniaco.

A conclusione di questo paragrafo, dedicato alle peculiarità della Costituzione bosniaca, occorre illustrare - seppure per sommi capi - una istituzione che non sembra trovare eguali in altri ordinamenti, almeno europei: la figura dell'Ufficio dell'Alto Rappresentante (OHR). L'Ufficio fu creato con l'Allegato X del General Framework Agreement for Peace, con il compito di attuare ed implementare gli aspetti civili del trattato di pace. La nomina dell'Alto Rappresentante avviene all'interno del Peace Implementation Council (PIC), organo internazionale costituito da cinquantacinque paesi ${ }^{14}$. La figura dell'Alto Rappresentante ${ }^{15}$ si caratterizza - ed ecco spiegata tale peculiarità - per essere al contempo un organo internazionale ed un organo dell'ordinamento interno della Bosnia. Infatti, quale organo internazionale è preposto a controllare ed incrementare l'applicazione degli aspetti civili dell'Accordo di Dayton, come sancito dall'Allegato X. Quale organo statale, grazie agli ulteriori poteri conferitigli dal PIC alla conferenza di Bonn ${ }^{16}$, può sostituirsi

\footnotetext{
${ }^{13}$ Queste sono: Convention on the Prevention and Punishment of the Crime of Genocide (1948); Geneve Convention IIV on the Protection of the Victims of War, and the 1977 Protocols I-II thereto (1949); Convention relating to the Status of Refugees and the 1966 Protocol thereto (1951); Convention on the Nationality of Married Women (1957); Convention on the Reduction of Statelessness (1961); International Convention on the Elimination of All Forms of Racial Discrimination (1965); International Convention on Civil and Political Rights and the 1966 and 1989 Optional Protocols thereto (1966); Covenant on Economic, Social and Cultural Rights (1966); Convention on the Elimination of All Forms of Discrimination against Women (1979); Convention against Torture and Other Cruel, Inhuman or Degrading Treatment or Punishment (1984); European Convention on the Prevention of Torture and Inhuman or Degrading Treatment or Punishment (1987); Convention on the Rights of the Child (1989); International Convention on the Protection of the Rights of All Migrant Workers and Members of Their Families (1990); European Charter for Regional or Minority Language (1992); Framework Convention for the Protection of National Minorities (1994).

${ }^{14} \mathrm{Fu}$ istituito tra 1'8 ed il 9 dicembre del 1995 con la prima conferenza di Londra, con l'obiettivo di vigilare e sostenere la stabilità del paese. Questo compito viene svolto dal braccio esecutivo del PIC, cioè il Comitato direttivo, costituito da: Canada, Francia, Germania, Russia, Italia, Giappone, Regno Unito, Stati Uniti, Presidenza dell'Unione Europea, Commissione Europea e Conferenza Islamica, rappresentata dalla Turchia. Il Comitato direttivo assume il compito di fornire le linee guida per l'operato dell'Alto Rappresentante.

${ }^{15}$ Ad oggi hanno ricoperto la carica di Alto Rappresentante: Carl Bildt, Carlos Westendorp, Wolfgang Petritsche, Paddy Ashdown, Christian Schwarz-Schilling, Miroslav Lajčak, Valentin Inzko.

${ }^{16}$ Con la conferenza di Bon, tenutasi nel dicembre del 1997, il Peace Implementation Council ha accordato all'Ufficio dell'Alto Rappresentante maggiori poteri, rispetto a quelli già previsti all'Allegato X del General Framework Agreement, al fine di superare l'ostruzionismo di alcune istituzioni locali nell'attuazione dell'accordo di pace. In particolare, all'Alto Rappresentante fu conferito il potere di sostituirsi alle istituzioni della Bosnia, sia locali che centrali, nell'applicazione degli accordi di pace, qualora non vi avessero provveduto direttamente queste. Inoltre, venne accordato l'ulteriore potere di rimuovere qualsiasi funzionario pubblico della Bosnia che avesse violato gli impegni o le condizioni degli accordi di Dayton. Entrambi questi poteri furono utilizzati dall'Alto Rappresentante, soprattutto nel periodo di attuazione della Costituzione: tra la fine degli anni Novanta dello scorso secolo e i primi anni Duemila. Si ricorda qui, in particolare, la destituzione dalla carica di Presidente della Repubblica serba di Nikola Poplašen, per il suo «operato palesemente in contrasto con i principi democratici e di rispetto dei diritti umani sanciti dall'Accordo di Dayton» (v. link: http://www.ohr/?p=55123). Nel 2001 fu destituito dalla carica di Presidente della Bosnia il membro croato, Ante Jelavić,
} 
a qualsiasi organo statale che non abbia provveduto autonomamente ad adeguarsi al dettato dell'Accordo di Dayton. La Corte costituzionale della Bosnia nella sentenza U 9/00 ${ }^{17}$ ha ritenuto l'esercizio di questo potere legittimo e non in contrasto con quanto sancito dalla Costituzione: più specificamente, il giudice costituzionale ha stabilito che non vi era stata violazione della Carta in quanto, nell'esercitare il suo potere di sostituirsi agli organi statali, l'Alto Rappresentante stava seguendo il principio della "doppia funzionalità" (funkcionalna dualnost): esterna ed interna. Per questo tutti gli atti adottati dall'Ufficio dell'Alto Rappresentante vanno considerati come atti di natura interna all'ordinamento bosniaco e come tali possono essere sindacati dalla Corte costituzionale.

Ad oggi, il potere dell'Alto Rappresentante di sostituirsi agli organi dello Stato bosniaco è stato utilizzato in modo limitato e solamente nella fase attuativa della Costituzione, quindi nel periodo immediatamente successivo all'entrata in vigore della Carta fino ai primi anni del Duemila.

\section{La gestione della diversità etnica a livello locale, ovvero del "federalismo etnico"}

È possibile comprendere il ruolo unico assunto dal fattore etnico nella costruzione dello Stato bosniaco e del suo ordinamento, solamente analizzando le disposizioni costituzionali riguardanti le istituzioni statali. Infatti, ogni istituzione, che rappresenti i poteri esecutivo, legislativo o giudiziario, deve assicurare la pari rappresentanza dei tre popoli costitutivi. A questo, si aggiunge anche un complesso sistema di veti che la Costituzione accorda ai rappresentanti dei tre gruppi etnici per impedire una possibile violazione dell'interesse vitale di un popolo costitutivo in sede legislativa o esecutiva. Tale istituto fu concepito dai costituenti al fine di impedire la messa in minoranza di un gruppo rispetto agli altri, in modo tale da ottenere delle decisioni assunte con il più largo margine di condivisione. Una tale concezione ha così portato all'instaurazione di una democrazia consociativa, quale «complex system of institution and systemic incentive structure that seek to achieve accommodation and stable democratic rule in severely divided societies ${ }^{18}$.

Infatti, l'importanza del ruolo assunto dal fattore etnico all'interno dell'ordinamento bosniaco emerge immediatamente dalla Carta costituzionale, in cui si sancisce l'esistenza di tre «popoli costitutivi» ${ }^{19}$ : i bosgnacchi, i croati ed i serbi, quali etnie maggioritarie all'interno del paese.

La presenza di questi tre gruppi costituisce l'ossatura di tutto il sistema costituzionale della Bosnia. Le stesse istituzioni statali e locali sono state concepite in modo tale da garantire la paritaria rappresentanza di ognuno dei tre gruppi a livello di esecutivo, legislativo e di potere giudiziario. Per questo, nel contesto dell'ordinamento bosniaco si parla di una vera e propria «istituzionalizzazione

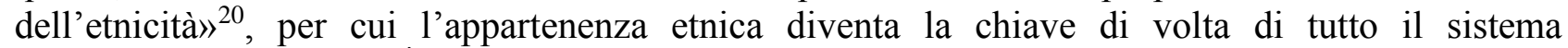
costituzionale del paese. È stato in questa ottica che la nuova Costituzione ha dato vita ad uno Stato federale che «shall consist of two Entities, the Federation of Bosnia and Herzegovina and the Republika Srpska» ${ }^{21}$. Tale forma di stato verticale aveva, da una parte, nelle intenzioni dei costituenti - sulla scorta del modello di power sharing di Lijphart ${ }^{22}$ - la finalità di lasciare un ampio spazio

per «attività sovversiva contro l'ordine costituito da Dayton e per il suo ostruzionismo radicale all'interno dell'istituzione statale di cui faceva parte» (v. link: http://www.ohr.int/?p=67244).

${ }^{17}$ Sentenza U 9/00 del 3 novembre 2000, consultabile al seguente link: http://www.ustavnisud.ba/admin//public/down/Bilten\%20USBiH\%202000_2.pdf

18 A. Merdzanović, Democracy by decree. Prospects and Limits of Imposed Consociational Democracy in Bosnia and Herzegovina, Ibidem-Verlag, Stuttgard, 2015, p. 113.

${ }^{19}$ Ultimo paragrafo del preambolo della Costituzione della Bosnia-Erzegovina.

${ }^{20}$ Cfr. R. C. Slye, The Dayton Peace Agreement: Constitutionalism and Ethnicity, in «Faculty Scholarship Series», n. 4426, 1996, p. 471; J. Woelk, La transizione costituzionale della Bosnia Erzegovina, op. cit., pp. 106-108.

${ }^{21}$ Art. I, comma 3, della Costituzione della Bosnia-Erzegovina.

${ }^{22}$ Cfr. A. Lijphart, Democracy in Plural Societies. A Comparative Exploration, Yale University Press, New Haven, 1977, pp. 25 passim. 
all'autonomia decisionale locale dei diversi gruppi. Dall'altra, invece, la soluzione federale sembrava essere quella più scontata per tenere unito un contesto in cui le divisioni si erano sempre più acuite in seguito al conflitto ed in cui la divisione territoriale in Enti federali risultava essere la conseguenza diretta delle conquiste militari. Nella sostanza dei fatti, quindi, i costituenti non hanno fatto altro che riconoscere e legittimare all'interno del paese le conseguenze territoriali del conflitto, pur sancendo all'articolo primo della Costituzione che «Bosnia and Herzegovina shall continue its legal existence under international law as a State [...] and with its present internationally recognized borders».

Ciò significa che lo Stato bosniaco non è una nuova creazione dal punto di vista del diritto internazionale, ma si pone in rapporto di continuità con la personalità giuridica della Repubblica di Bosnia-Erzegovina, con i medesimi confini riconosciuti dalla Comunità internazionale in seguito alla dichiarazione di indipendenza dalla Jugoslavia nel 1992. Ciò che cambia è la composizione interna del paese: adesso Stato federale formato da due Enti.

La Federazione di Bosnia-Erzegovina $(\mathrm{FBiH})$ fu costituita il 18 marzo del 1994, in seguito alla stipula dell'Accordo di Washington ${ }^{23}$ tra il Governo della Repubblica di Bosnia-Erzegovina e quello della Repubblica di Croazia, dietro forti pressioni dell'Amministrazione statunitense, al fine di porre fine alle ostilità tra i bosgnacchi ed i croato-bosniaci iniziate nel 1993. E con l'ulteriore obiettivo di compattare la situazione interna del paese in vista delle trattive per la pace che si sarebbero tenute da lì ad un anno. La Federazione di Bosnia-Erzegovina è costituita da dieci Cantoni.

L'altra Entità che compone lo Stato bosniaco è la Repubblica serba (RS), formatasi nel gennaio del 1992 in seguito alla volontà dei parlamentari serbo-bosniaci di opporsi all'indipendenza dalla Jugoslavia e di formare una entità territoriale separata dallo Stato bosniaco. La RS si contraddistingue, invece, per essere un ordinamento unitario. Per comprendere i rapporti che intercorrono tra lo Stato centrale e le entità, e con essi il riparto di competenze, è necessario prendere le mosse dall'articolo III della Costituzione in cui essi sono disciplinati. Il primo comma di tale articolo stabilisce quelle che sono le materie e le «responsibilities of the institutions of Bosnia and Herzegovina»: (a) la politica estera, (b) la politica commerciale estera, (c) la politica doganale, (d) la politica monetaria, (e) il finanziamento delle istituzioni e delle obbligazioni internazionali assunte dal Paese, (f) la politica e la regolazione delle questioni sull'immigrazione, i rifugiati e i richiedenti asilo politico, (g) l'applicazione delle leggi internazionali ed inter-Entità circa le norme di diritto penale, comprese le relazioni con l'Interpol, (h) l'istituzione ed il funzionamento dei mezzi di comunicazione comuni ed internazionali, (i) la regolazione dei rapporti tra le entità, (j) il controllo dello spazio aereo. A questo elenco di competenze esclusive dello Stato si può aggiungere, dal 2005, anche la materia della politica di difesa, in seguito alla creazione di una singola forza armata a livello statale. In base all'articolo I, comma settimo, della Costituzione anche la politica sulla cittadinanza statale appartiene in via esclusiva alle istituzioni dello Stato centrale. Inoltre, all'articolo III della Costituzione, comma quinto, vengono previste tre possibili diverse modalità per ampliare il novero delle competenze esclusive delle istituzioni dello Stato centrale, rispetto a quelle già espressamente previste in Costituzione.

In primo luogo, le Entità possono decidere, liberamente e di comune accordo, di cedere una parte, o più, delle competenze loro spettanti alle istituzioni statali. Per quanto riguarda le modalità con cui queste cessioni di competenza abbiano luogo occorre fare riferimento alla prassi, in quanto tanto la Costituzione che la legge rimangono silenti sul punto. In particolare, occorre analizzare gli unici tre casi in cui le Entità hanno raggiunto un accordo sulle ulteriori materie da cedere alla competenza statale: in materia di tassazione indiretta, la formazione di forze armate comuni per la difesa dello Stato e la fondazione dell'Alto Consiglio Giudiziario. Dalla prassi seguita è emerso che le parti dell'accordo non devono essere solo le entità, ma anche lo Stato, in quanto questo atto non solo produce nuove competenze per le istituzioni statali, ma anche degli obblighi, e per questo appare

\footnotetext{
${ }^{23}$ Il testo completo dell'Accordo è reperibile al seguente link: https:/www.usip.org/sites/default/files/file/resources/collections/peace_agreements/washagree_03011994.pdf.
} 
necessaria la presenza dello Stato quale parte dell'accordo, al fine di impegnarsi ad eseguirli. La sottoscrizione dell'accordo deve avvenire da parte dei Primi Ministri di ogni Entità, non prima però che le assemblee legislative abbiano approvato l'accordo. Inoltre, secondo l'interpretazione che la Corte costituzionale bosniaca ha fornito sull'articolo III/5 della Costituzione, solamente le competenze della FBiH e della RS possono essere trasferite allo Stato e non viceversa, in quanto le competenze dello Stato «appartengono esclusivamente alle istituzioni della Bosnia-Erzegovina e non esistono principi costituzionali in base ai quali queste possano essere trasferite alla competenza delle istituzioni delle Entità» ${ }^{24}$. Sempre secondo questa sentenza, non è possibile per le due Entità ritirare o negare il proprio consenso una volta che la cessione sia avvenuta, in quanto la materia oggetto di accordo non è più nella disponibilità degli stessi.

Una ulteriore modalità di ampliamento delle competenze statali, sempre previsto dall'articolo III/5 della Costituzione, è quello dell'assunzione da parte delle istituzioni centrali delle funzioni precedentemente esercitate da alcune istituzioni di carattere internazionale, create dall'Accordo di Dayton, una volta che il loro mandato in Bosnia sia terminato.

L'ultima modalità con cui le istituzioni statali possono ottenere l'ampliamento delle proprie competenze è quello previsto nel caso in cui «are necessary to preserve the sovereignty, territorial integrity, political independence and international personality of Bosnia and Herzegovina». Qualora uno di questi casi si dovesse materializzare concretamente e vi fosse la necessità di un ampliamento delle competenze dello Stato per farvi fronte, le istituzioni centrali dovranno seguire il principio di adeguatezza: adottando solamente quelle competenze necessarie che siano in grado di rispondere alla situazione concreta.

Al fine di comprendere il sistema di riparto delle competenze all'interno dell'ordinamento bosniaco occorre che la lettura dell'articolo III/1 della Costituzione avvenga in combinato disposto con l'articolo III/3.a, il quale sancisce che tutte le funzioni ed i poteri che non sono stati attribuiti espressamente dalla Costituzione alle istituzioni centrali dello Stato sono di competenza residuale delle Entità. Questo significa che i costituenti hanno attribuito maggiori competenze alla RS ed alla FBiH che alla Stato centrale. Infatti, le materie di competenza delle istituzioni statali sono circoscritte e devono sempre trovare un puntuale ancoramento nel testo costituzionale. Ne discende così un ampio margine di autonomia per quest'ultime. Confermato anche dalla possibilità per queste di «establish special parallel relationship with neighboring states» ${ }^{25}$, pur nel rispetto di «sovereignty and territorial integrity of Bosnia and Herzegovina» ${ }^{26}$.

L'ampio margine di autonomia di cui godono i due Enti federali è dimostrato anche dal fatto che questi possiedono proprie istituzioni autonomamente organizzate nell'ambito del potere legislativo, esecutivo e giudiziario. Il potere legislativo all'interno della Federazione di BosniaErzegovina è esercitato dalla propria Assemblea parlamentare, costituita da una Camera dei popoli e da una Camera dei rappresentanti (Costituzione FBiH, IV.A). Questa ultima Camera è costituita da 98 rappresentanti, eletti nelle sole circoscrizioni della Federazione e da cittadini ivi residenti per un mandato di quattro anni. Tra questi 98 parlamentari ve ne devono essere almeno quattro per popolo costitutivo, al fine di garantire una rappresentanza minima per ogni popolo costitutivo. La Camera dei popoli, invece, è composta da 58 parlamentari, di cui diciassette per ogni popolo costitutivo, a cui si aggiungono sette seggi riservati a coloro che non si definiscono appartenenti a nessun popolo costitutivo. I parlamentari di questa Camera vengono eletti in modo indiretto da parte delle Assemblee dei dieci Cantoni che costituiscono la Federazione di Bosnia-Erzegovina. La Camera dei popoli ha il precipuo compito di proteggere gli interessi dei popoli costitutivi in questa Entità.

\footnotetext{
24 Sentenza U 11/08 del 30 gennaio 2009, paragrafo 21; consultabile al seguente link: http://sup.ustavnisud.ba/public/down/Bilten_2009.pdf

${ }^{25}$ Art. III, comma 2, lettera a, della Costituzione della Bosnia-Erzegovina.

${ }^{26}$ Ibidem.
} 
Il potere esecutivo, invece, si caratterizza per essere esercitato dal Presidente della FBiH e dal Governo della FBiH (Costituzione FBiH, IV.B). Il Presidente della Federazione viene eletto dall'Assemblea parlamentare della Federazione di Bosnia-Erzegovina in seduta comune. Nel rispetto del principio della democrazia consociativa e del bilanciamento etnico, il Presidente deve nominare due suoi vice, che però devono appartenere a popoli costitutivi diversi tra loro e da quello di chi li ha nominati. Il mandato presidenziale ha una durata di quattro anni. Il Governo della Federazione è composto dal Premier, da due vicepremier e da sedici ministri, ognuno dei quali ha un sostituto, che non deve mai appartenere allo stesso popolo costitutivo del ministro, e la stessa regola vale per i due vice del Premier. I nomi di coloro che andranno a ricoprire i ministeri vengono designati sulla base del criterio della rappresentanza etnica proporzionale. Questo significa che otto ministri devono essere designati all'interno del gruppo dei bosgnacchi, cinque tra $\mathrm{i}$ croati e tre tra $\mathrm{i}$ serbi. Pur rimanendo in capo al Premier la prerogativa di nominare un ministro non appartenente a nessuno dei popoli costitutivi, al posto di uno dei ministri provenienti dall'etnia maggiormente rappresentata. I due vice del Premier vengono nominati tra i ministri che compongono il Governo. Il Gabinetto viene nominato dal Presidente della federazione, ma per entrare nel pieno delle sue funzioni deve ricevere la fiducia da parte dell'Assemblea parlamentare in seduta comune. Quanto al potere giudiziario nella Federazione di Bosnia-Erzegovina (Costituzione FBiH, IV.C) viene prevista l'esistenza di tribunali di primo grado a livello municipale e cantonale, che operano alternativamente a seconda dell'entità economica della contesa o della gravità del reato. Vi è poi una Corte suprema, in ogni capoluogo di Cantone, che assume il compito di tribunale d'appello e, infine, la Corte costituzionale della Federazione di Bosnia-Erzegovina che si esprime sulla costituzionalità degli atti nomativi prodotti nella Federazione rispetto alla sua Costituzione.

Per quanto riguarda la Repubblica serba il potere legislativo viene esercitato dalla sola Assemblea nazionale della RS (art. 71, Costituzione RS), pur essendo stata introdotta anche la Camera dei popoli, in seguito all'adeguamento rispetto alla sentenza U 5/98 della Corte costituzionale delle Bosnia, nota anche come "sentenza dei popoli costitutivi". L'Assemblea nazionale è composta da 83 delegati, eletti all'interno del solo territorio della RS. I delegati vengono eletti per un mandato di quattro anni. L'Assemblea è guidata da un Presidente che viene eletto all'interno della medesima; il Presidente a sua volta nomina da un minimo di due ad un massimo di quattro vicepresidenti, sempre nel rispetto del criterio della pari rappresentanza etnica. La Camera dei popoli, invece, è composta da 28 membri, di cui otto per ogni popolo costitutivo, a cui si affiancano quattro seggi riservati per coloro che non appartengono a questi tre gruppi. I suoi membri vengono eletti da parte dell'Assemblea nazionale, per un periodo di quattro anni. Questa Camera ha il compito di affiancare l'Assemblea nazionale in sede decisionale solamente quando viene apposto un veto per la difesa degli interessi vitali di uno dei popoli costitutivi e per quanto riguarda la votazione sugli emendamenti proposti alla Costituzione delle Repubblica serba.

Al vertice del potere esecutivo nella RS si trova il Presidente (art. 69, Costituzione RS), il quale la rappresenta e ne esprime l'unità. La sua elezione avviene in modo diretto, da parte dei cittadini residenti e iscritti nelle liste elettorali della Repubblica serba. Qui si può notare la differenza con la Federazione di Bosnia-Erzegovina, dove, l'elezione del Presidente avviene in modo indiretto da parte dell'Assemblea parlamentare di questa Entità in seduta comune. Il mandato presidenziale ha una durata di quattro anni, durante il quale viene coadiuvato da due vicepresidenti che devono appartenere a popoli costitutivi diversi. Il Governo della RS è composto dal Presidente del Governo e da sedici ministri, proprio come nella Federazione, solo che qui otto ministri devono appartenere al popolo costitutivo dei serbi, cinque a quello dei bosgnacchi e tre a quello dei croati. Tuttavia, il Presidente del Governo può decidere di nominare un ministro non appartenente ad uno dei tre popoli costitutivi, privando così i serbi di un seggio, in quanto popolo maggiormente rappresentato. La nomina del Presidente e dei ministri avviene ad opera del Presidente della RS. Mentre, la fiducia al Governo viene accordata dalla sola Assemblea nazionale. Per quanto riguarda il potere giurisdizionale nella RS esso è così diviso: Tribunale di primo grado (Osnovni Sud); i Tribunali 
distrettuali (Okružni Sud), che costituiscono i tribunali di secondo grado. Mentre, al vertice dell'ordinamento giudiziario della RS vi è il Tribunale supremo (Vrhovni Sud RS) e la Corte Costituzionale della Repubblica serba.

Detto questo, non si potrebbe concludere questo paragrafo dedicato all'assetto interno della Bosnia in rapporto al fattore etnico senza aver speso qualche parola per descrivere lo status particolareggiato del Distretto di Brčko. Infatti, la città di Brčko ed il suo Distretto si caratterizzano per costituire, da un lato, un'unità amministrativa autonoma sotto la sovranità dello Stato bosniaco e, dall'altro, un condominio tra le diverse Entità (Art. VI, comma 4, Costituzione della BosniaErzegovina). La ragione di tale status va ricercata nell'impossibilità da parte dei diversi soggetti in causa di accordarsi, durante le trattative di pace, a quale delle due Entità dovesse spettare la municipalità di Brčko, porto fluviale sulla Sava, di un certo interesse strategico per entrambi. Non riuscendosi a trovare un accordo, e per non impedire il raggiungimento della pace, le parti decisero di demandare la questione ad un apposito arbitrato internazionale. Il lodo, presentato il 5 marzo del 1999, sancì che la municipalità di Brčko dovesse costituire un'entità amministrativa autonoma sotto la sovranità dello Stato bosniaco. Così, oggi, il Distretto possiede un Governo, un'Assemblea parlamentare, un sistema giudiziario ed una forza di polizia propri, caratterizzati dal fatto di essere autonomi rispetto alle due Entità, pur rimanendo sotto la sovranità statale. Il potere legislativo viene esercitato dal Parlamento del Distretto di Brčko (art. 22, Statuto del Distretto di Brčko), composto da 31 parlamentari - di cui due devono essere necessariamente non appartenenti ai tre popoli costitutivi - che vengono eletti nella sola circoscrizione elettorale del Distretto. Dallo Statuto viene previsto, al fine di proteggere gli interessi vitali dei popoli costitutivi, un meccanismo di protezione contro la loro messa in minoranza durante le votazioni che abbiano per oggetto la modifica dello Statuto o del Regolamento del Parlamento (art. 33, Statuto). In questi casi è sempre necessario che la maggioranza che si esprime a favore delle modifiche comprenda al suo interno almeno un terzo dei parlamentari per ogni popolo costitutivo. Il potere esecutivo, invece, è esercitato dal Sindaco del Distretto, che viene eletto dal Parlamento per un mandato di quattro anni, coincidente con quello dell'organo che lo ha eletto. Il Sindaco a sua volta nomina chi presiede i Dipartimenti, che costituiscono una sorta di ministeri divisi per competenze (art. 47, Statuto). Il numero dei Dipartimenti può variare a seconda di quanto stabilito dal Sindaco, ma in ogni caso non possono raggiungere un numero superiore a dodici. Nello Statuto viene sancito che per la nomina dei capi di Dipartimento si debba seguire il criterio della composizione etnica paritaria, senza però indicare il numero esatto per ogni gruppo, lasciando quindi il bilanciamento nelle mani del Sindaco. Per quanto riguarda il potere giudiziario questo viene esercitato all'interno del Distretto dal Tribunale di primo grado e dal Tribunale d'appello. Il Distretto è privo di una Corte costituzionale, ragion per cui le questioni di legittimità sono demandate alla Corte costituzionale della Bosnia, come pure le questioni di attribuzione tra le istituzioni del Distretto e dello Stato (art. VI/2 Costituzione della Bosnia-Erzegovina).

\section{4. $\quad L$ ' istituzionalizzazione del fattore etnico all'interno dell'ordinamento bosniaco}

Per comprendere come il fattore etnico influisca in modo determinante anche sulla composizione e la struttura delle istituzioni statali della Bosnia, appare opportuno illustrarne gli aspetti peculiari, attraverso l'analisi del testo costituzionale. In particolare, facendo riferimento ai tre poteri: legislativo, esecutivo e giudiziario.

\subsection{Il potere legislativo}

Il Parlamento statale, disciplinato dall'articolo IV della Costituzione della Bosnia, è composto da due camere: la Camera dei Rappresentanti dell'Assemblea parlamentare della BosniaErzegovina (Predstanički Dom Parlamentarne Skupštine Bosne i Hercegovine), che ne costituisce la 
Camera bassa; e dalla Camera dei Popoli dell'Assemblea parlamentare delle Bosnia-Erzegovina (Dom Naroda Parlamentarne Skupštine Bosne i Hercegovine), che costituisce la camera alta dell'Assemblea parlamentare.

La Camera dei popoli è composta da quindici parlamentari, di cui dieci (cinque bosgnacchi e cinque croati) devono essere eletti dalla Camera dei popoli della Federazione di Bosnia-Erzegovina, rispettivamente dai parlamentari bosgnacchi e da quelli croati. Gli altri cinque rappresentanti serbi, invece, vengono eletti dall'Assemblea nazionale della Repubblica serba (art. IV/1.a, Costituzione $\mathrm{BiH}$ ). L'elezione di questa Camera non avviene, quindi, direttamente da parte del corpo elettorale, ma in modo indiretto, ad opera degli organi legislativi delle Entità. Questa situazione, nel periodo immediatamente successivo all'entrata in vigore della Costituzione, ha fatto supporre che gli interessi dei popoli costitutivi - che i parlamentari eletti sono chiamati a difendere - coincidessero con gli interessi dell'Entità da cui questi erano stati eletti. Tale prima interpretazione è stata smentita dalla sentenza U 5/98 - già citata quale "sentenza dei popoli" - che ha, invece, sancito l'eguaglianza dei popoli costitutivi su tutto il territorio dello Stato ${ }^{27}$, negando così l'identificazione di uno popolo costitutivo con una specifica Entità.

Il fattore etnico non solo rileva per quanto riguarda la composizione della Camera, ma anche rispetto ai suoi lavori. In particolare, perché sia raggiunto il quorum strutturale in questa Camera è necessaria la presenza di nove parlamentari, purché ve ne siano almeno tre per ogni popolo costitutivo (art. IV/1.b, Costituzione $\mathrm{BiH}$ ). Al suo interno la Camera si divide in: Club dei popoli, Collegio e Commissioni, in modo tale da facilitarne il funzionamento e accrescerne la qualità dei lavori. I Club dei popoli vengono formati dopo che i delegati hanno proceduto alla dichiarazione solenne di assunzione della carica e si dividono in tre $C l u b$, uno per popolo costitutivo (art. 4 del Regolamento della Camera dei popoli). Il Collegio è un organo interno della Camera, composto dal Presidente della Camera e dai suoi due vice, che ha il compito di riunirsi almeno una volta ogni due settimane e di fungere da tramite con l'altro ramo dell'Assemblea per il coordinamento dei lavori. Anche la formazione delle Commissioni parlamentari deve seguire il criterio della rappresentanza paritaria dei popoli costitutivi, infatti due terzi dei membri devono essere bosgnacchi e croati ed un terzo serbo.

La massima espressione della difesa degli interessi dei popoli costitutivi è rappresentata, tuttavia, dalla possibilità per i soli parlamentari della Camera dei popoli, quali difensori degli interessi dei popoli costitutivi, di apporre il veto rispetto ad un progetto di legge che debba essere messo ai voti. Infatti, l'articolo IV della Costituzione, al comma terzo, paragrafi (e) ed (f), prevede la possibilità per ogni delegato della Camera dei popoli di dichiarare una proposta di legge, o una parte di essa, lesiva (destruktivna) dell'interesse vitale del proprio popolo. In tale caso, si procede con la riunione del solo $C l u b$ a cui appartiene il delegato che abbia avanzato la dichiarazione di lesione dell'interesse vitale. Qualora il Club si esprima a favore della dichiarazione, il Presidente della Camera sarà costretto a nominare una commissione composta da un membro per ogni popolo costitutivo. La commissione ha il compito di ricercare una soluzione condivisa per risolvere la questione. Nel caso in cui, nell'arco di cinque giorni, la commissione congiunta non riesca a trovare una soluzione, la questione passa alla Corte costituzionale. La Corte attraverso una procedura ad hoc, definita "d'urgenza", dovrà fornire un parere motivato sulla questione, esprimendosi a favore o contro la reale esistenza di una lesione dell'interesse vitale del popolo costitutivo che ha sollevato la questione (art. IV/3.f Cost.). Se la Corte riconosce la natura lesiva del progetto di legge, o di una parte di esso, questo viene rinviato al suo proponente, che avrà l'obbligo di riformulare il progetto di legge, in modo tale da non ledere più l'interesse vitale. Invece, nel caso in cui la natura lesiva non venga riconosciuta dalla Corte, il progetto viene rimandato alla Camera che lo dovrà sottoporre ad una nuova votazione e su cui non sarà possibile apporre un veto di questo tipo.

L'esistenza di un tale istituto trova fondamento nella volontà di proteggere in massimo grado l'interesse dei popoli costitutivi, ma la particolare procedura che segue l'apposizione del veto, ed in particolare il peculiare ruolo della Corte costituzionale, si spiega con la ricerca di un giusto equilibrio

\footnotetext{
${ }^{27}$ Sentenza U 9/00 del 3 novembre 2000, paragrafo 34.
} 
tra le istanze dei popoli costitutivi ed il corretto funzionamento delle Camere. In quanto si temeva che la possibilità di apporre un veto potesse essere utilizzato dai delegati della Camera dei popoli con fini ostruzionistici.

Anche per quanto riguarda il quorum funzionale della Camera dei popoli sono previsti dei particolari correttivi per salvaguardare la massima condivisione delle scelte tra i popoli costitutivi. Infatti, perché una legge possa essere approvata da questa Camera è sufficiente la maggioranza semplice, ma all'interno di questa maggioranza devono essere necessariamente presenti i voti di almeno un terzo dei delegati per popolo costitutivo. Qualora in sede di votazione il numero necessario di voti per popolo costitutivo non venga raggiunto, il Presidente della Camera deve chiedere una pausa al fine di ricercare una soluzione condivisa con i $C l u b$ e riunire una apposita Commissione. Se dopo tre giorni non si è raggiunto un accordo, si procede con una nuova votazione in cui non è necessario raggiungere il quorum di un terzo per popolo costitutivo ${ }^{28}$.

L'altro ramo dell'Assemblea parlamentare è la Camera dei rappresentanti, formata da quarantadue membri, di cui ventotto eletti nelle circoscrizioni elettorali della Federazione di BosniaErzegovina e quattordici in quelle della Repubblica serba (art. IV/2.a Cost.). Anche la Camera dei rappresentanti ha un mandato di quattro anni e viene seguita al suo interno la medesima suddivisione interna in $C l u b$, Commissioni e Collegio; solo che qui i Club non vengono costituiti su base etnica, ma politica, come i gruppi parlamentari nel nostro ordinamento parlamentare. Il quorum strutturale nella Camera dei rappresentanti è fissato a 22 parlamentari. Le decisioni vengono raggiunte a maggioranza semplice, ma anche qui è previsto che tra $\mathrm{i}$ voti espressi dalla maggioranza ve ne sia almeno un terzo proveniente dai rappresentanti eletti in ogni Entità e non per popolo costitutivo. In caso contrario si applica la medesima procedura prevista per la Camera dei popoli. I rappresentanti di questa Camera non possiedono il diritto di veto, in quanto essi rappresentano i cittadini della Bosnia nel loro complesso e non gli interessi dei singoli popoli costitutivi.

Quando le Camere si riuniscono per la prima volta, dopo le elezioni, procedono all'elezione dei loro Presidenti e di due vicepresidenti, seguendo il principio della parità etnica. La presidenza è una carica che, una volta scaduto il mandato del primo presidente, viene occupata con un'alternanza periodica tra i due vicepresidenti, sino all'esaurimento del mandato quadriennale della Camera (art. IV/3.b Costituzione BiH). Qualsiasi decisione dell'Assemblea parlamentare deve essere prese da entrambe le Camere (art. IV/2.c), nel rispetto del principio del bicameralismo paritario vigente nel paese.

\subsection{Il potere esecutivo}

Al vertice del potere esecutivo si trova la Presidenza della Bosnia-Erzegovina (Predsjedništvo Bosne i Hercegovine), che si caratterizza per essere un organo collegiale composto da tre membri. In base a quanto previsto dall'articolo V della Costituzione, primo comma, la Presidenza è così costituita: un presidente appartenente al gruppo dei bosgnacchi ed uno a quello dei croati, i quali vengono eletti all'interno delle sole circoscrizioni elettorali della Federazione della $\mathrm{BiH}$; il terzo membro deve appartenere al popolo costitutivo dei serbi ed essere eletto nelle sole circoscrizioni della Repubblica serba (art. V/1.a Cost.). La carica di Presidente viene assunta inizialmente dal membro della Presidenza che alle elezioni abbi ottenuto il maggior numero dei voti, seguono poi a turno gli altri due membri, alternandosi così alla carica di Presidente ogni otto mesi fino all'esaurimento del mandato quadriennale (art. V/1.b Cost.).

Il quorum funzionale previsto per l'adozione delle decisioni in seno alla Presidenza cambia a seconda della materia oggetto della decisione. Infatti, la Costituzione all'articolo V, comma 2, paragrafo (b), prevede che per le seguenti materia la Presidenza debba esprimersi con un voto unanime dei suoi tre membri: «the foreign policy of Bosnia and Herzegovina; appointing ambassadors and other international representatives of Bosnia and Herzegovina; representing Bosnia and Herzegovina international and European organizations and institutions and seeking

${ }^{28}$ Art. IV, comma 3, paragrafo d, della Costituzione della Bosnia-Erzegovina. 
membership in such organizations and institutions of which Bosnia and Herzegovina is not a member; ratification of treaties; executing decisions of the Parliamentary Assembly» ${ }^{29}$. Qualora non venga raggiunto tale consenso, però, è possibile che la decisione venga assunta in seconda votazione con una maggioranza semplice dei membri della Presidenza.

Tale previsione, tuttavia, viene controbilanciata dalla possibilità di apporre un veto sospensivo da parte del membro della Presidenza dissenziente, il quale può dichiarare una delle materie sopra elencate lesiva per l'interesse vitale dell'Entità in cui è stato eletto (art. V/2.d Cost.). Una volta che il membro dissenziente abbia apposto il veto su di una questione, scatta l'obbligo per l'Assemblea nazionale della RS - qualora il membro dissenziente sia stato eletto sul territorio della Repubblica serba - di esprimersi sul veto. Allo stesso modo, qualora ad apporre il veto sia stato un membro eletto tra $\mathrm{i}$ bosgnacchi o i croati sarà, rispettivamente, il Club dei bosgnacchi o dei croati all'interno della Camera dei popoli della Federazione di Bosnia-Erzegovina ad esprimersi sul veto. Se anche le assemblee parlamentari delle Entità, nell'arco di dieci giorni dall'apposizione del veto, riconoscono l'esistenza di una lesione dell'interesse vitale dell'Entità, con una maggioranza qualificata dei due terzi, allora la decisione della Presidenza viene automaticamente disapplicata (art. V/2.d Cost.). La principale differenza che si può cogliere tra l'apposizione del veto all'interno della Camera dei popoli e all'interno della Presidenza risiede nel fatto che in quest'ultima il veto è rivolto alla protezione dell'interesse vitale dell'Entità in cui il membro della Presidenza è stato eletto e non del popolo costitutivo ${ }^{30}$. Nonostante ciò, si nota comunque l'esistenza di un criterio etnico, in quanto la votazione sulla proposta di veto avviene solo tra i parlamentari appartenenti allo stesso popolo costitutivo di chi ha sollevato la questione. Manifestandosi così per l'ennesima volta il pericoloso intreccio tra etnia ed Entità, che la Corte costituzionale aveva già respinto con la sentenza U 5/98 del 2000 .

Per quanto riguarda, invece, le materie espressamente previste dall'articolo $\mathrm{V}$, al terzo comma, paragrafi da (f) ad (i) della Costituzione, le decisioni vengono prese con la maggioranza semplice dei membri della Presidenza e non è possibile l'apposizione di nessun tipo di veto. Queste materie sono: «proposing, upon the recommendation of the Council of Ministers, an annual budget to the Parliamentary Assembly, reporting as requested, but not less than annually, to the Parliamentary Assembly on expenditures by the Presidency; coordinating as necessary with international and nongovernmental organizations in Bosnia and Herzegovina; performing such other functions as may be necessary to carry out its duties, as may be assigned to it by the Parliamentary Assembly, or as may be agreed by the Entities».

Anche il Consiglio dei ministri della Bosnia-Erzegovina (Vijeće ministara Bosne $i$ Hercegovine), come regolamentato dall'articolo $\mathrm{V}$ della Costituzione, quarto comma, segue la logica della rappresentanza paritaria dei popoli costitutivi. Infatti, questo si vede particolarmente bene nel momento della designazione dei ministri e dei viceministri. In primo luogo, una volta nominato il Presidente del Consiglio dei ministri da parte delle Presidenza, questi deve a sua volta nominare due vicepresidenti del Consiglio che devono necessariamente appartenere a due popoli costitutivi diversi tra loro e diversi da quello del Presidente (art. V/4.b Cost.). Allo stesso modo, la nomina dei ministri deve seguire un preciso equilibrio, stavolta non etnico ma territoriale, per cui non più di due terzi dei ministri deve essere stato eletto nelle circoscrizioni elettorali della Federazione di Bosnia-Erzegovina e non meno di un terzo in quelle della Repubblica serba (art. V/4.b Cost.). Ogni ministro deve nominare, a sua volta, due viceministri che debbono appartenere a popoli costitutivi diversi tra loro e dal suo. La durata in carica del Consiglio dei ministri è di quattro anni, a meno che il Presidente del Consiglio non si dimetta o venga sfiduciato dalla Camera dei rappresentanti.

\subsection{Il potere giudiziario.}

Neppure il vertice del potere giudiziario, la Corte costituzionale della Bosnia-Erzegovina (Ustavni Sud Bosne i Hercegovine), è stato esentato dalla logica dell'istituzionalizzazione

\footnotetext{
${ }^{29}$ Art. V, comma 3, paragrafi a-e, Costituzione della Bosnia-Erzegovina.

${ }^{30}$ N. Ademović, Ustavno pravo, Konrad Adenauer Stiftung, Sarajevo, 2012, p. 184.
} 
dell'etnicità e dalla paritaria rappresentazione dei popoli costitutivi. Infatti, la Corte costituzionale è composta da nove giudici, di cui quattro (due bosgnacchi e due croati) devono essere eletti dalla Camera dei rappresentanti della Federazione della Bosnia-Erzegovina; e due giudici appartenenti al popolo costitutivo dei serbi devono essere eletti all'interno dell'Assemblea della Repubblica serba. Ciò che caratterizza, però, la Corte costituzionale bosniaca è la presenza di ulteriori tre giudici che vengono nominati dal Presidente della Corte europea dei diritti dell'uomo, dopo che questi ha consultato la Presidenza della Bosnia-Erzegovina (art. VI/1.a Cost.). Inoltre, il testo della Costituzione specifica, all'articolo VI, comma 1, paragrafo $b$, che questi tre giudici per essere designati non devono possedere la cittadinanza della Bosnia-Erzegovina o quella di uno degli Stati confinanti (quali: Croazia, Serbia o Montenegro). La ratio della singolare presenza di tre giudici stranieri all'interno della Corte costituzionale bosniaca può essere reperita nella volontà dei costituenti di controbilanciare la presenza di possibili istanze etniche, in modo tale da impedire l'adozione di decisioni che vedessero la prevalenza di due popoli costitutivi su di uno solo, sancendone quini l'isolamento. Il fattore etnico viene nuovamente alla luce anche per quanto riguarda la struttura interna della Corte. Infatti, la Corte al suo interno deve nominare un Presidente e tre vicepresidenti, i quali devono appartenere a due popoli costitutivi diversi tra loro e da quello del Presidente, mentre la carica di terzo vicepresidente deve essere ricoperta da un giudice straniero.

Tra i consueti compiti che una corte costituzionale può assumere ve ne è uno del tutto particolare che svolge la Corte costituzionale bosniaca, cioè quello di esprimersi sull'apposizione del veto per presunta violazione degli interessi vitali di un popolo costitutivo da parte dei parlamentari della Camera dei popoli. Questo significa che la Corte assume anche, oltre le consuete attribuzioni, il compito di difendere e tutelare gli interessi dei popoli costitutivi, come sanciti dal testo della Costituzione. Infatti, qualora in seguito alla sollevazione del veto nella Camera dei popoli, non si riesca a raggiungere un accordo interno per sbloccare la soluzione di stallo creatasi, sarà la Corte ad intervenire. L'azione dinanzi la Corte viene portata dal presidente della Camera dei popoli, a cui segue l'avvio di una procedura d'urgenza con cui la Corte deve accertare, in prima istanza, se il veto sia stato correttamente sollevato rispetto ad una delle materie espressamente prevista dall'articolo IV/3 (a) - (f) della Costituzione. In caso di risposta affermativa, la Corte deve valutare se la questione verta realmente sull'interessa vitale di uno dei popoli costitutivi, inoltre, deve stabilire se sia stato leso realmente tale interesse rispetto alla questione oggetto del giudizio. Tale competenza della Corte costituzionale è stata definita anche come giudizio per lo "sblocco dell'Assemblea parlamentare", in quanto l'apposizione del veto spesso è venuta a coincidere con l'attività ostruzionistica di alcuni gruppi in Parlamento.

Per concludere questa parte, dedicata all'istituzionalizzazione del fattore etnico all'interno dell'ordinamento bosniaco, sembra opportuno ricordare anche il ruolo precedentemente assunto dai popoli costitutivi all'interno dei vertici della Banca centrale della Bosnia-Erzegovina. La Costituzione prevedeva infatti che, per i primi sei anni dalla sua entrata in vigore, il Consiglio direttivo della Banca centrale fosse composto da un Governatore non cittadino bosniaco e designato dal Fondo Monetario Internazionale, affiancato e coadiuvato da tre membri designati dalla Presidenza della BosniaErzegovina (art. VII/3 Cost.). I tre membri dovevano appartenere ad ognuno dei tre popoli costitutivi. In seguito alla scadenza del primo mandato del Consiglio direttivo della Banca centrale il criterio della rappresentanza etnica per la composizione del Consiglio è venuto meno.

\section{Limiti e futuro del costituzionalismo bosniaco}

Anche a distanza di venticinque anni dalla firma degli accordi di Dayton e dall'entrata in vigore della Costituzione non risulta semplice fornire un bilancio esaustivo sul costituzionalismo bosniaco, da una parte, a causa della complessità dell'ordinamento costituzionale ivi creato; dall'altra, 
in ragione della difficoltà di immaginare un nuovo sistema che sia diverso da quello etnico-nazionale che ormai pervade ogni aspetto dell'ordinamento statale.

In primo luogo, il maggior limite che sembra incontrare la struttura costituzionale del paese, come emersa in seguito all'Accordo di Dayton, è la stessa istituzionalizzazione del fattore etnico. Infatti, tra le originarie intenzioni dei costituenti vi era quella di favorire la cooperazione e la riappacificazione tra le tre principali etnie del paese, attraverso il riconoscimento di un loro status paritario all'interno dello Stato e delle sue istituzioni. Questo, nel lungo termine, ha portato ad una maggiore identificazione dei singoli con il proprio gruppo di appartenenza, a scapito di una concezione multiculturale e condivisa dello Stato. Portando spesso le conseguenze di questa situazione al parossismo, tanto da pregiudicare in più occasioni la stabilità dello Stato e delle sue istituzioni. Allo stesso tempo, il ruolo dei partiti etno-nazionalisti e delle loro istanze sembra aver trovato nuova linfa, dopo il conflitto, proprio nella divisione tra popoli costitutivi.

Inoltre, dal principio della paritaria rappresentanza dei tre popoli costitutivi, sembra invece emergere una posizione privilegiata di questi, se confrontata con quella assunta dagli Altri ${ }^{31}$, cioè quella macrocategoria di soggetti che non si identificano con uno dei popoli costituivi o che appartengono a qualche minoranza riconosciuta nel paese ${ }^{32}$, i quali non trovano una collocazione nella struttura statale. Infatti, per una persona che non si dichiari appartenente ad uno dei tre popoli costitutivi, il testo costituzionale impedisce indirettamente la candidatura e la possibilità di essere eletto quale delegato alla Camera dei popoli, alla Presidenza della Bosnia-Erzegovina o quale giudice della Corte costituzionale.

Questa particolare questione è emersa a cavallo del 2005 e 2006, in vista delle elezioni nazionale del 2006, quando il Sig. Sejdić, presidente della Comunità Rom della Bosnia, ed il Sig. Finci, presidente della Comunità ebraica della Bosnia, hanno chiesto alla Commissione elettorale della Bosnia-Erzegovina un parere circa la loro candidatura rispettivamente a delegato della Camera dei popoli ed alla Presidenza. La Commissione ha loro negato la possibilità di candidarsi, in quanto la Costituzione del paese prevede la necessaria appartenenza ad uno dei tre popoli costitutivi per poter ricoprire tali cariche. I due, vedendosi respinta la loro richiesta, hanno così fatto ricorso alla Corte EDU, la quale nel dicembre del 2009 ha constatato la violazione dell'articolo 14 della CEDU, in combinato disposto con l'articolo 3 del Protocollo n.1, da parte dello Stato bosniaco ${ }^{33}$.

Da questa sentenza emerge e viene confermato un importante limite insito nell'ordinamento costituzionale della Bosnia: una palese discriminazione tra i popoli costitutivi e coloro che invece non vi rientrano, cioè gli "Altri”. Ciò che ne emerge è un sistema costituzionale ideato e basato sull'ethnos, piuttosto che sul demos. A scapito del principio della cittadinanza, che si può definire "neutrale" in quanto prescinde dal criterio dell'appartenenza etnica ${ }^{34}$.

Questo, tuttavia, non è l'unico aspetto problematico emerso dall'ordinamento della Bosnia nel corso degli anni. Infatti, le complesse strutture previste dalla Costituzione al fine di bilanciare e proteggere l'aspetto etnico hanno prodotto un significativo rallentamento ed appesantimento delle istituzioni statali. Tanto che in più occasioni è stato registrato non solo un ritardo delle istituzioni statali rispetto ad importanti questioni per il paese, ma un loro vero e proprio blocco. Ciò è emerso particolarmente in sede legislativa, dove alcuni parlamentari hanno spesso fatto ricorso al veto etnico con il solo fine ostruzionistico, in modo tale da rallentare i lavori dell'Assemblea parlamentare

\footnotetext{
${ }^{31}$ L'ultimo paragrafo della Costituzione della Bosnia accanto ai popoli costitutivi riconosce l'esistenza degli Altri.

${ }^{32}$ La "Legge sulla difesa dei diritti delle minoranze della Bosnia-Erzegovina" è stata approvata il $1^{\circ}$ aprile del 2003, con 1'atto PS BiH br. 24/03. Con questo atto sono state riconosciute diciassette minoranze presenti in Bosnia, tra cui anche quella italiana, discendente da una comunità trentina insediatasi qui alla fine dell'Ottocento.

${ }^{33}$ L. E. Wakely, From Constituent Peoples to Constituent: Europe Solidifies Fundamental Political Rights for Minority Groups in Sejdić v. Bosnia, in «The North Carolina Journal of International Law and Commercial Regulation», n. 3, 2010, pp. 233-254.

${ }^{34}$ J. Woelk, La transizione costituzionale, op. cit., p. 263.
} 
quando questa era chiamata a decidere determinate questioni ritenute controverse per alcuni popoli costitutivi. Questa problematica è emersa in modo predominante durante il periodo immediatamente successivo all'entrata in vigore della Costituzionale, tanto che è dovuto intervenire (secondo il criterio della "doppia funzionalità") anche l'Alto Rappresentante per adottare le decisioni necessarie perché alcune istituzioni dello Stato potessero essere formate e così iniziare il proprio operato. Ad oggi, lo stesso processo per l'adesione all'Unione europea ed alla Nato è costantemente differito ed ostacolato attraverso l'uso di veti incrociati ed altri istituti previsti dalla Costituzione ${ }^{35}$ per l'adozione delle decisioni più importanti, non ultima la previsione costituzionale della necessaria unanimità tra $\mathrm{i}$ membri della Presidenza.

Altro aspetto problematico appare essere l'intreccio tra gli interessi del singolo gruppo etnico e l'interesse delle singole Entità. Una tale coincidenza, che tanto i costituenti quanto la stessa Corte costituzionale hanno negato, rispecchia le divisioni sorte in seguito alla guerra. In particolare, questo risulta essere una delle conseguenze della "pulizia etnica" operata nel corso della guerra, per cui il singolo gruppo etnico si identifica con 1'Entità in cui prevale numericamente ${ }^{36}$.

A tutto questo si deve aggiungere anche l'assenza di una visione comune e condivisa dello Stato da parte dei popoli costitutivi, i cui effetti si possono percepire anche in Costituzione. Infatti, il circoscritto novero dei poteri assegnato alle istituzioni statali e l'ampio margine di indipendenza concesso alle Entità rispetto allo Stato centrale impedisce la creazione di un'entità statale che si possa definire stabile. Causa di questo è anche il persistente intervento nelle questioni bosniache interne da parte delle confinanti Serbia e Croazia. Infatti, questi due Stati quali firmatari del General Framework Agreement, insieme alla Bosnia ed alla Comunità europea, si ponevano quali garanti della pace e della stabilità del paese. A questo si aggiunge anche l'esistenza di particolari rapporti intercorrenti tra le Entità ed i due paesi limitrofi, come previsto dall'articolo III della Costituzione, secondo comma, che non agevolano una visione comune sulle priorità dello Stato.

In conclusione, si può dire che il maggiore e forse più serio limite della odierna Costituzione bosniaca è stato quello di aver congelato ed accettato gli effetti del conflitto, senza aver tentato di ricostruire - o di fornire gli strumenti necessari per farlo - una società veramente in grado di essere plurale. Infatti, il particolare status dei popoli costitutivi, previsto in Costituzione, non ha creato uno Stato privo di discriminazioni, ma anzi ne ha accentuato gli aspetti di divisione, per cui oggi nel paese abbiamo cittadini di "prima categoria" in quanto appartenenti ai popoli costitutivi e cittadini di "seconda categoria" - gli Altri - che non godono degli stessi diritti, soprattutto nel campo dell'elettorato passivo.

Per non parlare, poi, dell'aspetto che suscita la maggiore preoccupazione, cioè quello dell'istituzionalizzazione della divisione per gruppi etnici. Oggi, nel paese ogni aspetto della vita istituzionale, ma anche di quella privata, sembra seguire il criterio dell'appartenenza etnica ${ }^{37}$.

Esposti qui solo alcuni dei principali limiti emersi dell'ordinamento costituzionale della Bosnia, appare difficile, se non presuntuoso, cercare di tracciare uno schizzo degli aspetti che potrebbero interessare il costituzionalismo bosniaco in futuro. Tuttavia, cercando di superare tali difficoltà sulla base degli elementi oggettivi disponibili, si può tentare di individuarne alcuni aspetti, primo fra tutti quello di una riforma costituzionale. La necessità di una revisione del testo costituzionale è emersa più volte nel corso della breve storia costituzionale della Bosnia. In particolare, nel 2006 con il così detto "pacchetto di aprile", bocciato per soli due voti in Assemblea parlamentare, si è quasi arrivati ad una importante riforma dell'assetto istituzionale del paese. Era stato previsto dal progetto di revisione costituzionale, infatti, un temperamento del principio etnico, con l'inclusione nelle istituzioni statali anche degli Altri. Inoltre, era stato previsto un uso molto più

\footnotetext{
${ }^{35}$ Come emerso dal report annuale (2020) della Commissione comune per l'integrazione europea. Consultabile al seguente link: https://www.parlament.ba/committee/read/39?commiteeMandate=298.

${ }^{36}$ Cfr. K. Trnka, Specifičnosti ustavnog uređenja, art. cit., p. 50.

${ }^{37}$ Cfr. A. Mujkić, Mi, građani etnopolisa, Šahinpašić, Sarajevo, 2007, p. 21.
} 
circoscritto del veto per la difesa degli interessi vitali dei popoli costitutivi, al fine di limitarne l'uso, spesso spregiudicato. Nonostante questo primo importante tentativo di revisione non sia riuscito altri ne sono seguiti, ma sempre con esito negativo. Questo può forse significare che la sfida futura più significativa per l'ordinamento bosniaco è quella di superare il costituzionalismo eteroimposto ed alcune soluzioni problematiche che sono derivata dall'Accordo di Dayton. Nonostante questo accordo abbia avuto l'importante merito di aver posto fine ad una guerra sanguinosa e di aver ideato un sistema costituzionale accettabile nel breve termine e di essere stato in grado di bilanciare le richieste di tre gruppi etnici, non è riuscito a creare un ordinamento privo di discriminazioni e realmente funzionale. Da questo, è emersa sempre più spesso la necessità di una riforma della Carta con cui ripensare il concetto di pluralismo all'interno della Bosnia, che possa garantire le tre maggiori etnie del paese, ma anche le altre minoranze, che fino ad ora hanno trovato scarsa protezione ed un ruolo estremamente limitato, se non a livello locale, nelle istituzioni statali. Quello che sembra sempre più necessario è la creazione di un ordinamento statale che sia basato sul fattore neutrale della cittadinanza e meno su quello dell' appartenenza etnica, che i fatti hanno dimostrato dividere piuttosto che unire la popolazione. Senza però con questo sconfessare interamente il sistema della democrazia consociativa e quindi anche la protezione accordata ai vari gruppi etnici della Bosnia. Inoltre, appare sempre più necessaria una Costituzione che sia in grado di essere il più inclusiva e condivisa possibile $^{38}$, in quanto «a Democratic State cannot successfully function without a strong support of citizens and among citizens, without a support from below (political parties, civil society, religious associations, intermediate bodies, as so on) and without the consent of broad cross-section of population ${ }^{39}$. Il tentativo di mitigare i correttivi già previsti per proteggere l'interesse dei gruppi etnici, invece, risulta necessario anche per lo stesso funzionamento dello Stato e delle sue istituzioni. Troppo spesso, infatti, nella pratica quotidiana delle maggiori istituzioni statali si sono avuti rallentamenti o vere e proprie situazioni di stallo dovute ad un uso improprio e spregiudicato dei veti etnici, con il solo fine di impedire il raggiungimento di un accordo sulle questioni connotate da una maggiore importanza e per questo, spesso, controverse.

Ma fin tanto che la società bosniaca non riuscirà a smarcarsi dalle divisioni che si porta dietro dalla conclusione della guerra come un'ombra, attraverso una revisione degli aspetti problematici dell'odierna Costituzione, sarà difficile la costruzione di un ordinamento realmente pluralistico ed in grado di operare senza il continuo supporto della Comunità internazionale.

\footnotetext{
${ }^{38}$ Questa necessità era stata evidenziata già nei primi anni Duemila e poi confermata nel 2004 dalle Risoluzioni del Consiglio d'Europa nn. 1383 e 1384.

${ }^{39}$ V. Piergigli, Bosnia-Herzegovina: in search for the constitutional identity?, in L. Benedizione, V. R. Scotti (a cura di), Twenty years after Dayton. The constitutional transition of Bosnia and Herzegovina, LUISS Academy, Roma, 2016, p. 128.
} 